\title{
On the Klein-Gordon oscillator subject to a Coulomb-type potential
}

\author{
K. Bakke* and C. Furtadd \\ Departamento de Fúsica, Universidade Federal da Paraíba, \\ Caixa Postal 5008, 58051-970, João Pessoa, PB, Brazil.
}

\begin{abstract}
By introducing the scalar potential as modification in the mass term of the Klein-Gordon equation, the influence of a Coulomb-type potential on the Klein-Gordon oscillator is investigated. Relativistic bound states solutions are achieved to both attractive and repulsive Coulomb-type potentials and the arising of a quantum effect characterized by the dependence of angular frequency of the Klein-Gordon oscillator on the quantum numbers of the system is shown.
\end{abstract}

PACS numbers: 03.65.Pm, 03.65.Ge, 03.30.+p

Keywords: Klein-Gordon oscillator, Coulomb-type potential, biconfluent Heun equation, relativistic bound states

*Electronic address: kbakke@fisica.ufpb.br

${ }^{\dagger}$ Electronic address: furtado@fisica.ufpb.br 


\section{INTRODUCTION}

In recent decades, the relativistic generalization of the harmonic oscillator has attracted a great deal of attention. The most known relativistic model of the harmonic oscillator was introduced by Moshinsky and Szczepaniak [1], which is known as the Dirac oscillator, and has been investigated by several authors [2-11]. In particular, the Dirac oscillator has attracted a great interest in studies of Jaynes-Cummings model [4, 8], the Ramsey-interferometry effect [9] and quantum phase transitions [10, 11]. On the other hand, a relativistic model of the harmonic oscillator has also been proposed for a scalar particle by Bruce and Minning [12] based on the Dirac oscillator [1]. Bruce and Minning [1] showed that an analogous coupling to the linear Dirac oscillator coupling can be introduced into the Klein-Gordon equation in such a way that one can recover the Schrödinger equation for a harmonic oscillator in the nonrelativistic limit. This coupling proposed by Bruce and Minning is known as the KleinGordon oscillator [12 15]. As example, by considering the isotropic Klein-Gordon oscillator in $(2+1)$ dimensions, the Klein-Gordon equation becomes:

$$
\left[\mathcal{E}^{2}-m^{2}\right] \phi=[\hat{p}+i m \omega \rho \hat{\rho}] \cdot[\hat{p}-i m \omega \rho \hat{\rho}] \phi
$$

where $m$ is the rest mass of the scalar particle, $\omega$ is the angular frequency of the KleinGordon oscillator, $\rho=\sqrt{x^{2}+y^{2}}$ and $\hat{\rho}$ is a unit vector in the radial direction. In recent

years, the Klein-Gordon oscillator has been investigated in noncommutative space [16, 17], in noncommutative phase space [18] and in $\mathcal{P} \mathcal{T}$-symmetric Hamiltonian [19].

Recently, several authors have shown the interest in investigating relativistic effects $\left\lfloor_{20}\right.$ 23] on systems where the motion of a particle is governed by harmonic oscillations, such as the vibrational spectrum of diatomic molecules [24], the binding of heavy quarks [25, 26] and the oscillations of atoms in crystal lattices, by mapping them as a position-dependent mass system [27-30]. The importance of these potentials arises from the presence of a strong potential field. In particular, Bahar and Yasuk [20] dealt with the quark-antiquark interaction as a problem of a relativistic spin-0 particle possessing a position-dependent mass, where the mass term acquires a contribution given by a interaction potential that consists of a linear and a harmonic confining potential plus a Coulomb potential term. It is worth mentioning other works that have explored the relativistic quantum dynamics of a scalar particle subject to different confining potentials which can be in the interest of several areas of physics $[31$ 36]. 
The aim of this work is to study the influence of a Coulomb-type potential on the KleinGordon oscillator. In recent years, the confinement of a relativistic scalar particle to a Coulomb potential has been discussed by several authors [37-41]. As discussed in Ref. [41], the procedure in introducing a scalar potential into the Klein-Gordon equation follows the same procedure in introducing the electromagnetic 4-vector potential. This occurs by modifying the momentum operator $p_{\mu}=i \partial_{\mu}$ in the form: $p_{\mu} \rightarrow p_{\mu}-q A_{\mu}(x)$. Another

procedure was proposed in Ref. [42] by making a modification in the mass term in the form: $m \rightarrow m+S(\vec{r}, t)$, where $S(\vec{r}, t)$ is the scalar potential. This modification in the mass term has been explored in recent decades, for instance, by analysing the behaviour of a Dirac particle in the presence of static scalar potential and a Coulomb potential [43] and a relativistic scalar particle in the cosmic string spacetime [44]. In this work, we investigate the influence of a Coulomb-type potential on the Klein-Gordon oscillator by introducing the scalar potential as modification in the mass term in the Klein-Gordon equation. We obtain bound state solutions to the Klein-Gordon equation for both attractive and repulsive Coulomb-type potentials and show a quantum effect characterized by the dependence of angular frequency of the Klein-Gordon oscillator on the quantum numbers of the system, which means that not all values of the angular frequency are allowed.

The structure of this paper is as follows: in section II, we study the Klein-Gordon oscillator subject to a Coulomb-type potential in the Minkowski spacetime in $(2+1)$ dimensions; in section III, we present our conclusions.

\section{KLEIN-GORDON OSCILLATOR UNDER THE INFLUENCE OF A COULOMB-TYPE POTENTIAL}

In this section, we study the behaviour of the Klein-Gordon oscillator subject to a Coulomb-type potential in $(2+1)$ dimensions. We consider the cylindrical symmetry, then, we write the line element of the Minkowski spacetime in the form (with $c=\hbar=1$ ):

$$
d s^{2}=-d t^{2}+d \rho^{2}+\rho^{2} d \varphi^{2}
$$

Thereby, the Klein-Gordon equation describing the interaction of the Klein-Gordon oscillator and static scalar potential is given by (with $c=\hbar=1$ )

$$
[m+S(\rho)]^{2} \phi=-\frac{\partial^{2} \phi}{\partial t^{2}}-[\hat{p}+i m \omega \rho \hat{\rho}] \cdot[\hat{p}-i m \omega \rho \hat{\rho}] \phi
$$


where $S(\rho)$ is a scalar potential, $m$ is the rest mass of the scalar particle, $\omega$ is the angular frequency of the Klein-Gordon oscillator, $\hat{p}=-i \vec{\nabla}, \rho=\sqrt{x^{2}+y^{2}}$ and $\hat{\rho}$ is a unit vector in the radial direction. In this way, by considering a Coulomb-type potential

$$
S(\rho)=\frac{f}{\rho}= \pm \frac{|f|}{\rho}
$$

where $f$ is a constant, the Klein-Gordon equation (3) becomes

$$
-\frac{\partial^{2} \phi}{\partial t^{2}}+\frac{\partial^{2} \phi}{\partial \rho^{2}}+\frac{1}{\rho} \frac{\partial \phi}{\partial \rho}+\frac{1}{\rho^{2}} \frac{\partial^{2} \phi}{\partial \varphi^{2}}+m \omega \phi-m^{2} \omega^{2} \rho^{2} \phi-m^{2} \phi-\frac{2 m f}{\rho} \phi-\frac{f^{2}}{\rho^{2}} \phi=0 .
$$

In what follows, we shall be considering particular stationary solutions to Eq. (5) that are eigenfunctions of the operator $\hat{L}_{z}=-i \partial_{\varphi}$. In this way, we have

$$
\phi=e^{-i \mathcal{E} t} e^{i l \varphi} R(\rho)
$$

where $l=0, \pm 1, \pm 2, \ldots$. Then, substituting (6) into Eq. (5) , we obtain

$$
\frac{d^{2} R}{d \rho^{2}}+\frac{1}{\rho} \frac{d R}{d \rho}-\frac{\gamma^{2}}{\rho^{2}} R-\frac{2 m f}{\rho} R-m^{2} \omega^{2} \rho^{2} R+\beta^{2} R=0,
$$

where we have defined the following parameters in Eq. (7):

$$
\begin{aligned}
& \beta^{2}=\mathcal{E}^{2}-m^{2}+m \omega \\
& \gamma^{2}=l^{2}+f^{2}
\end{aligned}
$$

From now on, let us consider $\xi=\sqrt{m \omega} \rho$, thus, we rewrite the radial equation (7) in the form:

$$
\frac{d^{2} R}{d \xi^{2}}+\frac{1}{\xi} \frac{d R}{d \xi}-\frac{\gamma^{2}}{\xi^{2}} R-\frac{\delta}{\xi} R-\xi^{2} R+\frac{\beta^{2}}{m \omega} R=0
$$

where we have defined a new parameter

$$
\delta=\frac{2 m f}{\sqrt{m \omega}}
$$

Let us discuss the asymptotic behaviour of the possible solutions to Eq. (9), which are determined for $\xi \rightarrow 0$ and $\xi \rightarrow \infty$. From Refs. [44-46], the behaviour of the possible solutions to Eq. (9) at $\xi \rightarrow 0$ and $\xi \rightarrow \infty$ allows us to write the function $R(\xi)$ in terms of an unknown function $H(\xi)$ as it follows:

$$
R(\xi)=\exp \left(-\frac{\xi^{2}}{2}\right) \xi^{\sigma|\gamma|} H(\xi)
$$


where $\sigma= \pm 1$. Note that one should have in mind the possibility of existing a singular solution to Eq. (91). As pointed out in Refs. [46, 47], $\sigma=1$ yields a non-singular solution whereas $\sigma=-1$ yields a singular solution to Eq. (9). In this contribution, we consider only non-singular solutions to Eq.(9), that is, $\sigma=1$. Then, substituting the radial wave function given in Eq. (11) into Eq. (91), we obtain

$$
\frac{d^{2} H}{d \xi^{2}}+\left[(2|\gamma|+1) \frac{1}{\xi}-2 \xi\right] \frac{d H}{d \xi}+\left[\frac{\beta^{2}}{m \omega}-2-2|\gamma|-\frac{\delta}{\xi}\right] H=0 .
$$

The second order differential equation (12) corresponds to the Heun biconfluent equation [44, 48-50] and the function $H(\xi)$ is the Heun biconfluent function

$$
H(\xi)=H\left(2|\gamma|, 0, \frac{\beta^{2}}{m \omega}, 2 \delta, \xi\right) .
$$

Proceeding with our discussion about bound states solutions, let us use the Frobenius method [51, 52]. Thereby, the solution to Eq. (12) can be written as a power series expansion around the origin:

$$
H(\xi)=\sum_{j=0}^{\infty} a_{j} \xi^{j}
$$

Substituting the series (14) into (2), we obtain the following recurrence relation:

$$
a_{j+2}=\frac{\delta}{(j+2)(j+1+\alpha)} a_{j+1}-\frac{(\theta-2 j)}{(j+2)(j+1+\alpha)} a_{j},
$$

where $\alpha=2|\gamma|+1$ and $\theta=\frac{\beta^{2}}{m \omega}-2-2|\gamma|$. By starting with $a_{0}=1$ and using the relation (15), we can calculate other coefficients of the power series expansion (14). For instance,

$$
\begin{aligned}
a_{1} & =\frac{\delta}{\alpha} \\
& =\frac{2 m f}{\sqrt{m \omega}} \frac{1}{(2|\gamma|+1)} \\
a_{2} & =\frac{\delta^{2}}{2 \alpha(1+\alpha)}-\frac{\theta}{2(1+\alpha)} \\
& =\frac{2 m f^{2}}{\omega} \frac{1}{(2|\gamma|+1)(2|\gamma|+2)}-\frac{\theta}{2(2|\gamma|+2)}
\end{aligned}
$$

It is well-known that the quantum theory requires that the wave function (6) must be normalizable. Therefore, we assume that the function $R(\xi)$ vanishes at $\xi \rightarrow 0$ and $\xi \rightarrow \infty$. This means that we have a finite wave function everywhere, that is, there is no divergence of the wave function at $\xi \rightarrow 0$ and $\xi \rightarrow \infty$, then, bound state solutions can be obtained. 
However, we have written the function $H(\xi)$ as a power series expansion around the origin in Eq. (14). Thereby, bound state solutions can be achieved by imposing that the power series expansion (14) or the Heun biconfluent series becomes a polynomial of degree $n$. In this way, we guarantee that $R(\xi)$ behaves as $\xi^{|\gamma|}$ at the origin and vanishes at $\xi \rightarrow \infty$ [45, 46]. Through the recurrence relation (15), we can see that the power series expansion (14) becomes a polynomial of degree $n$ by imposing two conditions [44-46, 49, 50, 52 -54]:

$$
\theta=2 n \quad \text { and } \quad a_{n+1}=0,
$$

where $n=1,2,3, \ldots$. From the condition $\theta=2 n$, we can obtain:

$$
\mathcal{E}_{n, l}^{2}=m^{2}+2 m \omega_{n, l}\left[n+|\gamma|+\frac{1}{2}\right] .
$$

Hence, Eq. (18) is a general expression for the relativistic energy levels of the KleinGordon oscillator subject to a Coulomb-type potential, where we have coupled this scalar potential as a modification of the mass term in the Klein-Gordon equation. In contrast to the results of Ref. [12], we have that the influence of the Coulomb-like potential makes that the ground state to be defined by the quantum number $n=1$ instead of the quantum number $n=0$. Note that we have written the angular frequency $\omega$ in terms of the quantum numbers $\{n, l\}$ in Eq. (18). From the mathematical point of view, this dependence of the angular frequency of this relativistic oscillator on the quantum numbers $\{n, l\}$ results from the fact that the exact solutions to Eq. (12) are achieved for some values of the Klein-Gordon oscillator frequency. From the quantum mechanics point of view, this is an effect which arises from the influence of the Coulomb-type potential on the Klein-Gordon oscillator.

Henceforth, let us discuss this behaviour of the Klein-Gordon oscillator frequency. First of all, we must note that, at first glance, the result obtained in Eq. (18) shows that the relativistic energy levels do not depend on the parameter $\delta$ which gives rise to the Coulomb-type potential (defined in Eq. (10)). However, we need to analyse the second condition established in Eq. (17), that is, the condition $a_{n+1}=0$. By analysing this second condition, we obtain an expression involving specific values of the angular frequency of the Klein-Gordon oscillator and the parameter $\delta$. It is worth mentioning other analyses which have been made in recent years, as example, a relation involving the harmonic oscillator frequency, the Lorentz symmetry-breaking parameters and the total angular momentum quantum number obtained in Ref. [53], a relation involving a coupling constant of a Coulomb-like potential, 
the cyclotron frequency and the total angular momentum quantum number in semiconductors threaded by a dislocation density obtained in Ref. [49], and a relation involving the mass of a relativistic particle, a scalar potential coupling constant and the total angular momentum quantum number achieved in Ref. [44].

Thereby, let us assume that the angular frequency of the Klein-Gordon oscillator $\omega$ can be adjusted in such a way that the condition $a_{n+1}=0$ is satisfied in order that a relation between the angular frequency of the Klein-Gordon oscillator and the Coulomb-type potential parameter can be obtained. The meaning of achieving this relation is that not all values of the angular frequency $\omega$ are allowed, but some specific values of $\omega$ which depend on the quantum numbers $\{n, l\}$. For this reason, we label

$$
\omega=\omega_{n, l}
$$

Therefore, the conditions established in Eq. (17) are satisfied and a polynomial solution to the function $H(\xi)$ given in Eq. (14) is achieved [44 46, 50]. As an example, let us consider $n=1$, which corresponds to the ground state, and analyse the condition $a_{n+1}=0$. For $n=1$, we have $a_{2}=0$. The condition $a_{2}=0$, thus, yields

$$
\omega_{1, l}=\frac{2 m f^{2}}{(2|\gamma|+1)} .
$$

In this way, substituting Eq. (20) into Eq. (17), the expression of the energy level ( for $n=1)$ becomes:

$$
\begin{aligned}
\mathcal{E}_{1, l} & = \pm \sqrt{m^{2}+2 m \omega_{1, l}\left[n+|\gamma|+\frac{1}{2}\right]} \\
& = \pm m\left[1+4 f^{2} \frac{\left(|\gamma|+\frac{3}{2}\right)}{(2|\gamma|+1)}\right]^{1 / 2}
\end{aligned}
$$

In what follows, let us consider the simplest case of the function $H(\xi)$ which corresponds to a polynomial of first degree. In this way, for $n=1$, we can write

$$
H_{1, l}(\xi)=1+\frac{\delta}{\alpha} \xi
$$

Thereby, from Eq. (22), thus, Eq. (11) can be written in the form: $R_{1, l}(\xi)=$ $e^{-\xi^{2} / 2} \xi^{|\gamma|}\left(1+\frac{\delta}{\alpha} \xi\right)$.

Hence, the effects of the Coulomb-type potential introduced by a coupling with the mass term on the spectrum of energy of the Klein-Gordon oscillator correspond to a change of the 
energy levels, where the ground state is defined by the quantum number $n=1$. Moreover, a quantum effect characterized by the dependence of angular frequency of the Klein-Gordon oscillator on the quantum numbers $\{n, l\}$ of the system arises, whose meaning is that not all values of the angular frequency are allowed. In this way, the conditions established in Eq. (17) are satisfied and a polynomial solution to the function $H(\xi)$ given in Eq. (14) is achieved in agreement with Refs. [44 46, 50].

\section{CONCLUSIONS}

We have studied the influence of a Coulomb-type potential on the Klein-Gordon oscillator and shown that the ground state of the Klein-Gordon oscillator is defined by the quantum number $n=1$ instead of the quantum number $n=0$. Other quantum effect obtained is the dependence of angular frequency of the Klein-Gordon oscillator on the quantum numbers $\{n, l\}$ of the system, whose meaning is that not all values of the angular frequency are allowed. As an example, we have calculated the angular frequency of the ground state $n=1$ and obtained the expression of the energy level of the ground state.

It is worth mentioning that we have introduced the scalar potential as a modification of the mass term of the Klein-Gordon equation. However, as discussed in Ref. [41], one can use the same procedure of introducing the electromagnetic 4-vector potential by modifying the momentum operator as $p_{\mu} \rightarrow p_{\mu}-q A_{\mu}(x)$. By dealing with a Coulomb-type potential via a minimal coupling, new interesting results associated with the Klein-Gordon oscillator can be obtained. Moreover, the interaction between a relativistic scalar particle and the Klein-Gordon oscillator can be of interest in studies of the quark-antiquark interaction [20], position-dependent mass systems [27-30], the Casimir effect [55, 56] and the Kaluza-Klein theory [57].

\section{Acknowledgments}

The authors would like to thank CNPq (Conselho Nacional de Desenvolvimento Científico 
e Tecnológico - Brazil) for financial support.

[1] M. Moshinsky and A. Szczepaniak, J. Phys. A: Math. Gen. 22, L817 (1989).

[2] V. M. Villalba, Phys. Rev. A 49, 586 (1994).

[3] D. Itô, K. Mori, E. Carrieri, Nuovo Cimento A 51, 1119 (1967).

[4] P. Rozmej and R. Arvieu, J. Phys. A 32, 5367 (1999).

[5] K. Bakke, Gen. Relativ. Gravit. 45, 1847 (2013).

[6] K. Bakke and C. Furtado, Ann. Phys. (NY) 336, 489 (2013).

[7] J. Carvalho, C. Furtado and F. Moraes, Phys. Rev. A 84, 032109 (2011).

[8] A. Bermudez, M. A. Martin-Delgado and E. Solano, Phys. Rev. A 76, 041801(R) (2007).

[9] A. Bermudez, M. A. Martin-Delgado and A. Luis, Phys. Rev. A 77, 033832 (2008).

[10] A. Bermudez, M. A. Martin-Delgado and A. Luis, Phys. Rev. A 77, 063815 (2008).

[11] A. Bermudez, M. A. Martin-Delgado and E. Solano, Phys. Rev. Lett. 99, 123602 (2007).

[12] S. Bruce and P. Minning, Nuovo Cimento A 106, 711 (1993).

[13] V. V. Dvoeglazov, Nuovo Cimento A 107, 1413 (1994).

[14] N. A. Rao and B. A. Kagali, Phys. Scr. 77, 015003 (2008).

[15] A. Boumali, A. Hafdallah and A. Toumi, Phys. Scr. 84, 037001 (2011).

[16] B. Mirza, R. Narimani and S. Zare, Commun. Theor. Phys. 55, 405 (2011).

[17] M.-L. Liang and R.-L. Yang, Int. J. Mod. Phys. A 27, 1250047 (2012).

[18] Y. Xiao, Z. Long and S. Cai, Int. J. Theor. Phys. 50, 3105 (2011).

[19] J.-Y. Cheng, Int. J. Theor. Phys. 50, 228 (2011).

[20] M. K. Bahar and F. Yasuk, Advances in High Energy Physics, vol. 2013, Article ID 814985, 6 pages, 2013. Doi:10.1155/2013/814985.

[21] R. Kumar and F. Chand, Phys. Scr. 85, 055008 (2012).

[22] S. H. Dong, Int. J. Theor. Phys. 39, 1119 (2000).

[23] S. H. Dong, Int. J. Theor. Phys. 40, 559 (2001).

[24] S. M. Ikhdair, Int. J. Mod. Phys. C 20, 1563 (2009).

[25] C. Quigg and J. L. Rosner, Phys. Rep. 56, 167 (1979).

[26] M. Chaichian and R. Kögerler, Ann. Phys. (NY), 124, 61 (1980).

[27] L. Dekar, L. Chetouani and T. F. Hammann, Phys. Rev. A 59, 107 (1999). 
[28] A. D. Alhaidari, Phys. Rev. A 66, 042116 (2002).

[29] A. D. Alhaidari, Phys. Lett. A 322, 72 (2004).

[30] L. Serra and E. Lipparini, Europhys. Lett. 40, 667 (1997).

[31] A. de Souza Dutra and C.-S. Jia, Phys. Lett. A 352, 484 (2006).

[32] W.-C. Qiang, R.-S. Zhou and Y. Gao, Phys. Lett. A, 371, 201 (2007).

[33] A. S. de Castro, Phys. Lett. A 338, 81 (2005).

[34] A. D. Alhaidari, H. Bahlouli and A. Al-Hasan, Phys. Lett. A 349, 87, (2006).

[35] F. Domingues-Adame, Phys. Lett. A 136, 175 (1989).

[36] Y. Xu, S. He and C.-S. Jia, Phys. Scr. 81, 045001 (2010).

[37] Q. Wen-Chao, Chinese Phys. 12, 1054 (2003).

[38] H. Motavalli and A. R. Akbarieh, Mod. Phys. Lett. A 25, 2523 (2010).

[39] F. Yasuk, A. Durmus and I. Boztosun, J. Math. Phys 47, 082302 (2006).

[40] A. L. Cavalcanti de Oliveira and E. R. Bezerra de Mello, Class. Quantum Grav. 23, 5249 (2006).

[41] W. Greiner, Relativistic Quantum Mechanics: Wave Equations, 3rd Edition (Springer, Berlin, 2000).

[42] H. G. Dosch, J. H. Jansen and V. F. Müller, Phys. Norv. 5, 2 (1971).

[43] G. Soff, B. Müller, J. Rafelski and W. Greiner, Z. Naturforsch. A 28, 1389 (1973).

[44] E. R. Figueiredo Medeiros and E. R. Bezerra de Mello, Eur. Phys. J. C 72, 2051 (2012).

[45] A. Verćin, Phys. Lett. B 260, 120 (1991).

[46] J. Myrhein, E. Halvorsen and A. Verćin, Phys. Lett. B 278, 171 (1992).

[47] J. Grundberg, T. H. Hansson, A. Karlhede and J. M. Leinaas, Mod. Phys. Lett. B 5, 539 (1991).

[48] A. Ronveaux (ed.), Heun's differential equations (Oxford University Press, Oxford, 1995).

[49] K. Bakke and F. Moraes, Phys. Lett. A 376, 2838 (2012).

[50] K. Bakke, Ann. Phys. (NY) 341, 86 (2014).

[51] G. B. Arfken and H. J. Weber, Mathematical Methods for Physicists, sixth edition (Elsevier Academic Press, New York, 2005).

[52] C. Furtado, B. G. C. da Cunha, F. Moraes, E. R. Bezerra de Mello and V. B. Bezerra, Phys. Lett. A 195, 90 (1994).

[53] K. Bakke and H. Belich, Eur. Phys. J. Plus 127, 102 (2012). 
[54] K. Bakke and H. Belich, Ann. Phys. (NY) 333, 272 (2013).

[55] N. R. Khusnutdinov and M. Bordag, Phys. Rev. D 59, 064017 (1999).

[56] H. F. Mota and K. Bakke, Phys. Rev. D 89, 027702 (2014).

[57] C. Furtado, F. Moraes and V. B. Bezerra, Phys. Rev. D 59, 107504 (1999). 